Erhard Geißler: Drosophila oder die Versuchung. Ein Genetiker der DDR gegen Krebs und Biowaffen, Berlin (Berliner Wissenschafts-Verlag) 2010.

Viele Aspekte der Zeit des Kalten Krieges sind inzwischen in Büchern und wissenschaftlichen Aufsätzen aufgearbeitet worden. Das gilt auch für die internationalen Bemühungen um die Kontrolle der Biowaffen, für die wissenschaftliche Arbeit und der internationale Austausch unter Wissenschaftlern große Bedeutung hatten.

Aber was diesen Veröffentlichungen häufig fehlt ist das menschliche Flair, ist das Gefühl für historische Zufälle, die Bedeutung von Persönlichkeiten, und nicht zuletzt deren Einbettung in gesellschaftliche Vorstellungen und Systeme. Die Erinnerungen von Ehrhard Geißler bieten hier viele interessante Einblicke unter anderem auch in das Leben eines Wissenschaftlers in der DDR und nach der deutschen Einheit.

Erhard Geißler wurde 1930 geboren und studierte in den frühen 1950er Jahren in Leipzig Biologie. Früh blitzte sein Potenzial als experimenteller Molekularbiologe auf; früh auch sein eigener Kopf. Er eckte mehrfach wegen nicht-konformer Meinungen an, so weil er zu einer Zeit, als dies in der DDR-Fachwissenschaft nicht mehr opportun war, Lehrmeinungen der Lyssenkoschen Vererbungslehre vertrat. Um ihm die Erkenntnisse der Genetik näher zu bringen, wurde ihm nahegelegt, seine Diplomarbeit über die Fruchtfliege Drosophila zu schreiben. Das förderte nicht nur sein Interesse an der Genetik, sondern eröffnete ihm auch Türen in den Westen Deutschlands. Als einer der wenigen Wissenschaftler in der DDR baute er gute Verbindungen zu führenden Forschern in Westdeutschland im rasch wachsenden Feld der Genetik auf, und noch vor dem Mauerbau konnte er in Köln und in Westberlin bei Gastaufenthalten den aktuellen Stand der Genforschung kennenlernen.

Mit diesem Wissen gerüstet war Geißler zwischen 1965 und 1971 Professor für Genetik und Institutsdirektor an der Universität Rostock; danach bis 1987 Professor und Abteilungsleiter am Zentralinstitut der DDR für Molekularbiologie in Berlin. Ein wesentliches Arbeitsfeld war dabei die experimentelle Krebsforschung. Nach 1987 und bis zum Ende der DDR leitete er am Zentralinstitut eine kleine Gruppe von Wissenschaftlern, die sich mit Biowaffenkontrolle befassten eine Arbeit, die er, mit Unterstützung durch die VolkswagenStiftung, von 1992 bis 2000 an dessen Nachfolgeinstitution, dem Max-Delbrück-Zentrum für Molekulare Medizin in Berlin, fortführen konnte.

Geißler berichtet detailreich über die Schwierigkeiten eines international renommierten Wissenschaftlers in der DDR, über den Mangel an experimentellem Gerät, oder Bespitzelungen und willkürliche Eingriffen „von oben“. Ein Gutteil trug er allerdings auch selbst zu seinen Problemen bei: durch sein Streben nach wissenschaftlicher Unabhängigkeit und kritischer Reflektion über die eigene Wissenschaft. Gleichzeitig machte ihn seine Kreativität für die Fachwelt in der DDR, und gelegentlich auch für die politische Führung, interessant. Einige Veröffentlichungen über Genetik machten ihn zu einem der führenden Wissenschaftsethiker in der DDR, ohne dass er zuvor viel über Ethik nachgedacht hatte. Aber sie brachten ihn in Kontakt mit Kollegen aus anderen Disziplinen, in der DDR und darüber hinaus. Geißler begann, die Kühlungsborner Symposien zu organisieren, von deren intellektueller Qualität er im Buch begeistert berichtet.

Ein Thema, in dem die Problematik der wissenschaftlichen Fortschritte in seinem Fachgebiet besonders zum Ausdruck kommt, beschäftigte ihn seit den späten 1960er Jahren in zunehmendem Maße, nämlich die Biowaffen. Offiziell seit 1971 mit der Biowaffenkonvention international geächtet, gab es immer wieder Vermutungen, dass weiter an Biowaffen gearbeitet würde oder zumindest in Zukunft gearbeitet werden könnte. Die Entwicklung der Genetik eröffnete hierzu neue Möglichkeiten. Zunächst rezipierte Geißler vor allem die Literatur zu diesem Thema - die östliche wie die westliche. Nicht zuletzt auf der Grundlage seiner guten Erfahrungen mit dem internationalen Austausch unter Wissenschaftlern begann er sich um stärkere internationale Vernetzung mit Kolleginnen und Kollegen in anderen Ländern zu bemühen.

Dafür bekam er Unterstützung aus dem westlichen Ausland. Das Stockholm In- ternationale Peace Research Institute (SIPRI) hatte in den 1970er Jahren ein großangelegtes Projekt zur Geschichte der chemischen und biologischen Waffen durchgeführt. Anfang der 1980er Jahre war im SIPRI der Wunsch aufgekommen, sich mit den neueren Entwicklungen im Umfeld von Biowaffen zu befassen und dies mit einem Ost-West-Kooperationsprojekt zu verbinden. Erste Kontakte wurden zu Geißler, einem der wenigen ausgewiesenen Experten aus dem Osten, geknüpft.

Geißler war interessiert, für zwei Jahre nach Stockholm zu gehen. Das SIPRI war von seiner Expertise beeindruckt. Aber Abteilungen der Staatssicherheit hatten Bedenken, ob der politischen Zuverlässigkeit Geißlers. Zudem sollten, wie Geißler aus dem Studium seiner Akte in der Gauck-Behörde erfahren konnte, die Kreise eines Stasi-Spitzels nicht gestört werden. Geißler vermutet, dass der nach dem Ende der DDR als informeller Mitarbeiter der Stasi enttarnte ToxikologieProfessor Karlheinz Lohs dieser Spitzel war, ohne es beweisen zu können. Lohs war in den frühen 1980er Jahren Mitglied des Aufsichtsrats von SIPRI und häufiger dort zu Gast. Schließlich wurde in Berlin entschieden, dass die Kooperation mit dem SIPRI politisch wichtig sei; Geißler wurde gestattet, in einer Serie von kürzeren Aufenthalten das Projekt in Stockholm durchzuführen. Das Ergebnis war ein Sammelband unter dem Titel „Biological and Toxin Weapons Today“.

Aufbauend auf den Erkenntnissen dieser Arbeit und mit Hilfe seiner weiter gewachsenen internationalen Kontakte begann Geißler 1987 von Berlin aus an einem neuen Projekt unter dem Titel "Vaccines for Peace" zu arbeiten. Ziel war die internationale Vernetzung von Biowissenschaftlern, um die Transparenz in der Forschung und deren Ausrichtung auf gesellschaftlich relevante Aufgaben zu verbessern. Zwar gelang es Geißler, breite Unterstützung zu erhalten, z.B. auf der Ebene der Vereinten Nationen, aber es gab auch viel Widerstand, sowohl bei westlichen als auch bei östlichen Staaten. Was Geißler zu diesem Zeitpunkt noch nicht wusste: In der Sowjetunion war auch nach 1971 in großem Maßstab weiter an Biowaffen gearbeitet worden. Das „Biopreparat“-Programm endete erst 1992. 
Nach dem Ende der DDR blieb Geißler der Thematik trotz einiger Schwierigkeiten treu. Sein internationales Prestige als einer der führenden Experten auf dem Gebiet der Biowaffen verschaffte ihm ausreichende finanzielle Mittel, um weitere Forschung durchzuführen. So arbeitete er zur Konversion der Einrichtungen zur B-Waffen-Forschung in der Sowjetunion und schrieb mehrere Bücher zur Geschichte der Biowaffen.

Die Erinnerungen von Geißler erlauben einen intimen Einblick in das Wissenschaftsgeschehen in der DDR. Sie zeigen die vielen Widersprüche und Grautöne, oft mit einem altersmilden, schmunzelnden Unterton. Vor allem warnt Geißler davor, Vorurteile kritiklos zu übernehmen, und plädiert dafür, sich stets sein eigenes Urteil zu bilden. Dies gelte für die DDR, aber genauso auch für die Rüstungskontrolle, wie Geißler mit Verweis auf die „Massenverschwindungswaffen" des Irak bemerkt.

\section{Michael Brzoska}

Ines Lehmann, Die Außenpolitik der DDR 1989/1990. Eine dokumentierte Rekonstruktion, Baden-Baden (Nomos Verlag) 2010.

Etwas mehr als zwei Jahrzehnte nach der Vereinigung im Herbst 1990 legt Ines Lehmann eine kommentierte Dokumentation einer wichtigen Etappe der jüngeren deutschen Geschichte einer interessierten Öffentlichkeit vor. In geradezu akribischer Weise hat die Autorin das beeindruckende Puzzle bekannter, aber auch zahlreicher bisher unveröffentlichter Belege zu den außenpolitischen Prozessen im Vorfeld und während der Vereinigung der beiden deutschen Staaten zu einem die Begleitumstände der damaligen Zeit sehr erhellenden Mosaik zusammengefügt. Es mag erstaunen, dass es bisher keine umfangreiche Dokumentation über die außenpolitischen Entscheidungsprozesse in beiden deutschen Staaten in den Jahren 1989 und 1990 gegeben hat, und noch mehr, dass es so lange gedauert hat, die einzelnen Teile zusammenzutragen. Allerdings: Der außenpolitische Dienst der ehemaligen DDR wurde nach dem 3. Oktober 1990 fast vollständig abgewickelt und seine Beamten hatten - auch wegen des Vorgehens dabei - kaum gesteigertes Interesse, zur Aufklärung von
Vorgängen beizutragen, die aus ihrer Sicht unter Umständen gegen sie verwendet werden und ihnen dadurch persönliche Nachteile verschaffen konnten. Die lange Zeit vorwiegend auf Schuldzuweisung fokussierte Aufarbeitung der Politik der DDR - mithin auch ihrer Außenpolitik - verwandelte diese zudem, so Ines Lehmann ernüchtert, zu einem „verminten Gelände“, in dem differenzierte Betrachtungsweisen weder gefragt noch in der Forschung gefördert wurden. Umso mehr ist die Beharrlichkeit der Autorin zu würdigen, die sich allen Widrigkeiten zum Trotz und unter Verwendung erheblicher Eigenmittel ans Werk machte und tatsächlich ein solches hervorgebracht hat. Die Dokumentation ist auch deshalb so bedeutsam wie schwierig in ihrer Entstehung, weil sie praktisch ausschließlich das Agieren der letzten nicht demokratischen und der ersten demokratisch legitimierten Regierungen der DDR untersucht. Beide Regierungen standen unter enormem Zeit- und Problemdruck, die sorgsame Archivierung ihrer handlungsleitenden Dokumente war in der Zeit der ersten Regierung kaum gewollt, in der Zeit der zweiten kaum möglich. Eine Fülle von Entscheidungen und vor allem ihrer Vorbereitung wurden nicht hinreichend dokumentiert und konnten nur durch Zeitzeugen belegt werden - sofern diese aufgefunden werden konnten und willens waren, über die damalige Zeit Auskunft zu geben. Dutzende von Befragungen hat Ines Lehmann ergänzend zu ihrer mühevollen Kleinarbeit in deutschen Archiven geführt, und ihre Arbeit wäre nicht von Erfolg gekrönt worden, hätte sie sich allein auf öffentliche Archive beschränken müssen, zu denen ihr sogar in einzelnen Fällen der Zugang verweigert wurde. Obwohl sie zum Teil gezwungen war, auch auf Sekundärquellen zurückzugreifen, ergibt sich in der Summe ein beindruckend umfassendes Bild über ein besonders schwieriges Kapitel deutscher Geschichte, an dessen Zustandekommen der Autorin bleibender Verdienst gebührt.

Das Buch zeichnet nach, wie der Versuch der Regierung Modrow zwischen Dezember 1989 und März 1990 gescheitert ist - und scheitern musste, mithilfe einer für die vorhergehenden Verhältnisse unvorstellbar radikalen politischen Kombination aus Versuchen zur gleichzeitigen de- mokratischen Öffnung, ökonomischen Krisenmanagements und politischer Konsolidierung die DDR als eigenständigen Staat und alternatives Gesellschaftsmodell zu retten. Die DDR war nicht zu retten. Ihre einzige Existenzberechtigung bezog sie aus dem Vorhandensein einer anderen gesellschaftlichen Ordnung. In dem Maße, wie diese Ordnung de-legitimiert wurde, verschwand auch die Legitimation für den Fortbestand eines zweiten deutschen Staates. Man mochte in der damals noch machtausübenden politischen Klasse darauf setzen, dass für den Fortbestand der DDR auch das Interesse der östlichen Nachbarn, vor allem der Sowjetunion, an der deutschen Teilung als stabilisierendes Element der europäischen Sicherheit, maßgeblich sein würde. In Moskau bestimmten das politische Lagebild jedoch bereits sehr grundsätzliche, die eigene sowjetische Existenz betreffende, Sorgen. Die damalige sowjetische Führung war nicht mehr fähig, die Abstimmung mit den Füßen nun auch in der DDR aufzuhalten und sie schien willens, die Veränderungen bei den ehemaligen Satelliten zu nutzen, um die eigene Verankerung im europäischen Raum zu erhalten, vielleicht sogar auszubauen. Ihre Diplomatie fuhr zunehmend zweigleisig, wobei der für sie maßgebliche Akteur weniger in Berlin als in Bonn verortet wurde. Entsprechende Signale wurden im Spätherbst 1989 jedoch entweder nicht zur Kenntnis genommen oder aus Ratlosigkeit verdrängt. Die Spielräume für die Modrow-Regierung wurden in der Folge unerträglich schmal. Innenpolitisch an die Kette der Entscheidungen am „Runden Tisch“ gelegt, außenpolitisch an den Rand gedrängt, wurde die Regierung schleichend entmachtet, verkam zur Hülle politischer Prozesse, die sie selbst nicht mehr selbst in den Händen hielt. Ein außenpolitisches Konzept hatte sie nicht, und die Opposition in der DDR zeigte an einer erneuerten Außenpolitik wenig Interesse. Am „Runden Tisch“ wurden außenpolitische Fragen kaum behandelt, Verteidigungs- und sicherheitspolitische Aspekte wurden an „Katzentischen" diskutiert und spielten für die große Reformpolitik keine wirklich wichtige Rolle. Die Regierung Modrow mochte vielleicht noch einige Notenblätter in ihren Händen halten, die Musik wurde jedoch nicht mehr von ihr gespielt. Kaum ein anderes Ereignis machte 
dies deutlicher als der gänzlich verpuffte Versuch Hans Modrows, mit einem eigenen Vorschlag zur Bildung einer Konföderation die Initiative zurückzugewinnen. Die Modrow-Regierung hatte keinerlei Verhandlungsmacht und schließlich auch kein Mandat mehr, in eigener Verantwortung zu verhandeln - nach der Verkündung des 10-Punkte-Plans von Helmut Kohl und innenpolitisch nach der widerstandslosen Erstürmung der Berliner Zentrale des Ministeriums für Staatsicherheit war endgültig klar, dass nicht nur die Tage der Modrow-Regierung, sondern auch die Tage der DDR gezählt waren.

Der Außenpolitik der ersten demokratisch legitimierten Regierung nach den Volkskammerwahlen vom 18. März 1990 widmet Ines Lehmann viel Aufmerksamkeit. Zur Gründungsgeschichte der Regierung gehört ein Wahlkampf, der emotional aufgeheizt und von nationalen Tönen stark beeinflusst worden war und deren unterlegte Absicht, mit dem Wahlergebnis den Weg zur deutschen Einheit zu ebnen, der damaligen „Allianz für Deutschland“ einen kaum so erwarteten, überwältigenden Sieg bescherte. Die Weichenstellung hin zur deutschen Einheit beließ die Initiative in Bonn, wo sie schon in den vergangenen Monaten gelegen hatte, und reduzierte die Aufgabe für die deutsche Außenpolitik im Wesentlichen auf das Ziel, möglichst genehme Rahmenbedingungen für die vorgesehene Vereinigung zu gewährleisten. Allerdings tickten die Uhren in Bonn und Berlin in den ersten Monaten nicht sofort synchron. Mit der demokratischen Legitimation ausgestattet, suchten die Reformkräfte des Wendeherbstes ihre politischen Vorstellungen in den politischen Prozess einzubringen. Anders als jene, die in der Vereinigung eine Erweiterung des Westens sahen, verfolgte die Regierung DeMaiziere eine außenpolitische Linie, welche die Überwindung der deutschen Teilung als Chance für die Überwindung der europäischen Spaltung verstand - und umgekehrt. Sie war lange Zeit dabei der Illusion verhaftet, auf Augenhöhe handeln zu können und als gleichberechtigt anerkennt zu werden. Vor allem im Außenministerium unter Leitung Markus Meckels - aber auch im Ministerium für Abrüstung und Verteidigung - wurden Konzepte entwickelt, die gesamteuropäische und zugleich mitteleuropäische Alternativen zur Sicherheit der Blöcke bilden sollten. Ernsthaft um Interessenausgleich bemüht, wurden einige der Vorschläge vorschnell des Dilettantismus geziehen und dadurch ihrer potenziellen Substanz als Ideenspender für eine alternative Europapolitik beraubt. Die Revolutionäre von 1989 mussten durchaus schmerzlich erfahren, dass nicht die Überzeugungskraft einer durchdachten Idee, sondern vielmehr die Durchsetzungskraft von handfesten Interessen darüber entscheidet, was letztlich politisch erreicht oder nicht erreicht werden kann. Als die 2+4-Verhandlungen (die vier Siegermächte des Zweiten Weltkriegs sowie die beiden deutschen Staaten) begannen, schien man sich, wie Ines Lehmann zu Recht vermutet, offenbar nicht klar über die eigenen begrenzten Möglichkeiten zu sein. Die auf am Verhandlungstisch unterbreiteten Vorschläge aus dem Hause Meckel stießen unter den machtpolitischen Verhältnissen auf Unverständnis und wurden von den westlichen Alliierten als Versuch angesehen, die sowjetischen Interessen zu bedienen. Schon deshalb wurde ihnen wenig Interesse zuteil, denn der eigentliche Partner saß für sie unter den bestehenden Vorzeichen in Moskau - nicht in Berlin. Die Koalitionsregierung geriet zunehmend zwischen die Mühlsteine widerstreitender Interessen zwischen CDU und SPD über Ziele und Wege zur deutschen Vereinigung. Vor allem die CDU stand unter starkem politischen Druck, den Prozess der Vereinigung zu beschleunigen und das Fenster der Möglichkeiten zu nutzen. Zunehmend war unklar, ob die Sowjetunion noch imstande sein würde, den Vereinigungsprozess konstruktiv zu begleiten und außenpolitisch handlungsfähig zu bleiben. Ines Lehmann zeigt im Detail auf, unterstützt vor allem durch Informationen aus dem Privatarchiv von Markus Meckel, wie sich die Differenzen innerhalb der Regierung zunehmend auf die Gestaltungsfähigkeit auswirkten, bis schließlich das Auseinanderbrechen der Regierung unausweichlich wurde. Sie zeigt dabei aber auch, wie stark diese Prozesse äußerer Einflussnahme durch die Bonner Parteien unterlagen.

Für die kurze Zeit vom Mauerfall bis zur Vereinigung, so bilanziert die Autorin, hatten sich die Regierungsverantwor- tung tragenden Politiker außen- und sicherheitspolitisch viel vorgenommen. $\mathrm{Zu}$ viel, wie sich zeigen sollte, zu viel, weil sie nicht über die Kraft und die Unterstützung verfügten, um die selbst gesteckten Ziele zu erreichen. Es bleibt das Verdienst der Verwalter der untergehenden DDR, zum friedlichen Verlauf der deutschen Vereinigung Wichtiges beigetragen zu haben. Der Prozess der Auflösung und Abwicklung der DDR - von Ines Lehmann ebenso sachkundig wie schonungslos beschrieben - wird retrospektiv in Sonntagsreden zumeist als eine der größten Leistungen der deutschen Geschichte gewürdigt. Es gibt aber auch Stimmen, die behaupten, dass Deutschland einfach Glück hatte. Das Verdienst dieses Buches ist, dass es lebendig und sichtbar werden lässt, wie historische Umwälzungen zustande kommen - von Menschen gemacht, oft von Zufällen abhängig, von Interessen getrieben und von mehr oder weniger weisen Entscheidungen begünstigt. Als Lehrbuch für ein besseres Verständnis der deutschen Geschichte in Europa ist dies durchaus kein geringer Lohn. 129 Euro, die der Verlag seinen Leserinnen und Lesern hierfür abverlangt, sind allerdings ein stolzer und leider auch abschreckender Preis.

\section{Hans J. Gießmann}

Simon Menz, Die Verantwortlichkeit der Mitarbeiter privater Militär- und Sicherheitsunternehmen nach Art. 8 ICC-Statut. Zugleich ein Beitrag zum Sonderdeliktscharakter von Kriegsverbrechen, Beiträge zum Internationalen und Europäischen Strafrecht, Band 9, Berlin (Verlag Duncker \& Humblot) 2011.

Mit dem Kalten Krieg endete zwar der Ost-West-Konflikt, jedoch stieg die Zahl regionaler Konflikte, die andersartige militärische Herausforderungen und zugleich ein neues Berufsfeld schufen: die Arbeit in privaten Militär- und Sicherheitsfirmen, die die regulären Armeen in ihren Tätigkeiten unterstützen. Mitarbeiter dieser privaten Firmen wurden in den letzten Jahren zunehmend in bewaffnete Auseinandersetzungen verwickelt, in deren Zuge sie Kriegsverbrechen begangen haben sollen, so etwa während des Irakkriegs. Diese Entwicklung stellt den Ausgangspunkt der vorliegenden Dissertation dar, die untersucht, inwie- 
fern dieses ,private‘ Personal einer völkerstrafrechtlichen Verantwortung für Kriegsverbrechen unterliegt. Mit dieser Fragestellung reiht sich das Werk in die aktuelle wissenschaftliche Diskussion über rechtliche Probleme der Privatisierung bewaffneter Konflikte ein. Der Arbeit liegt die These zu Grunde, dass die Mitarbeiter innerhalb einer völkerrechtlichen Grauzone agieren, sodass sie an das für reguläre Kombattanten geltende Kriegsvölkerrecht nicht gebunden und damit für Kriegsverbrechen nicht zur Verantwortung zu ziehen sind. Rechtliche Grundlage der Untersuchung ist Art. 8 ICC-Statut mit seiner umfassenden Tatbestandsdefinition von Kriegsverbrechen.

Das erste Kapitel führt in die Aufgaben und Tätigkeiten der Sicherheits- und Militärunternehmen ein und legt so die Grundlage für das Verständnis der Arbeit. Das zweite Kapitel fragt nach dem völkerrechtlichen Status der Mitarbeiter privater Sicherheitsfirmen innerhalb bewaffneter Konflikte, um so Rückschlüsse auf ihre etwaige völkerstrafrechtliche Verantwortlichkeit zu ziehen. Menz unternimmt auf Grundlage der Haager Landkriegsordnung, den Genfer Abkommen (GA I-IV), dem I. Zusatzprotokoll (ZP I) und dem Völkergewohnheitsrecht einen Versuch der Differenzierung: Auf Seiten der Akteure unterscheidet er zwischen Kombattant, Zivilist, Söldner und dem illegitimen bzw. Quasi-Kombattanten, hinsichtlich des Einsatzgebiets unterscheidet er zwischen internationalem und nicht-internationalem bewaffnetem Konflikt. In Bezug auf die Akteure kommt er unter Prüfung der Voraussetzungen der Art. 4 GA III und Art. 43, 44 ZP I zu dem Ergebnis, dass die Mitarbeiter privater Sicherheitsfirmen im Regelfall weder den De-iure- noch den De-facto-Kombattantenstatus besitzen. Menz schließt nach der Negativdefinition des Art. 50 ZP I und den weiteren Ausführungen des Art. 51 ZP I auf einen Zivilistenstatus, weist den die Truppe begleitenden Mitarbeitern (Art. 4 A Abs. 4 GA III) aber einen Kriegsgefangenenstatus $\mathrm{zu}$, der sie normalerweise von der Teilnahme an Feindseligkeiten ausschließt. Daneben schließt er den Söldnerstatus aus, da im Regelfall die eng gefassten Voraussetzungen des Art. 47 ZP I nicht erfüllt werden. Die Rechtsfigur des illegitimen Kombattanten scheide eben- falls aus, u.a. weil diese dem kodifizierten Vertragsrecht fremd sei. Die Einordnung als Quasi-Kombattant sei auf Grundlage des Art. 4 A Abs. 4 GA III nicht zu rechtfertigen. Für den nicht-internationalen Konflikt weist Menz den Mitarbeitern unter Verweis auf die nicht erfüllten Voraussetzungen der Art. 43, 44 ZP I ebenfalls einen Zivilistenstatus zu. In einem abschließenden Unterkapitel attestiert er Kriegsverbrechen einen Sonderdeliktscharakter, da ,als Täter [...] nur solche Personen in Betracht kommen, die eine Verbindung zu einer Konfliktpartei aufweisen“ (S. 144).

Das dritte Kapitel untersucht auf Grundlage des Art. 8 ICC-Statut, der „praktisch relevanteste[n] Sanktionsnorm für Kriegsverbrechen" (S. 144), die individuelle völkerstrafrechtliche Verantwortlichkeit. An Hand der objektiven Voraussetzungen des Art. 8 - und auch der subjektiven des Art. 30 ICC-Statut - prüft Menz den Charakter publik gewordener „privat“ begangener Straftaten. Er schlussfolgert, dass diese als Kriegsverbrechen nach Art. $8 \mathrm{zu}$ werten seien und damit aus materiell-rechtlicher Sicht vom ICC verfolgt werden könnten. Daneben stellt Menz fest, dass diese Verfolgung aber in der Praxis durch die Zuständigkeitsproblematik (Nachrangigkeit des ICC gegenüber der nationalen Strafverfolgung) und politische Rahmenbedingungen (etwa die Nichtanerkennung des ICC durch die USA) erschwert werde. Insgesamt widerlegt Menz in diesem Kapitel die vorangestellte These und attestiert so den Mitarbeitern trotz Zivilistenstatus eine Bindung an das Kriegsvölkerrecht - die Zugehörigkeit zu einer Konfliktpartei vorausgesetzt (eine Beauftragung falle unter diese Zugehörigkeit). Nach dieser Prüfung der Hauptthese untersucht der Autor die Verantwortlichkeit des privaten Sicherheitsunternehmens als juristische Person und schließt, dass diese nicht existiert, da der ICC nur Straftaten natürlicher Personen ahnden kann. Hinsichtlich der Geschäftsführung kommt Menz zu dem Schluss, dass Art. 28 ICCStatut die Basis bietet, diese zur Verantwortung zu ziehen. Um haftbar zu sein, müsse das Unternehmen generell hierarchisch strukturiert sein. Eine Anklage werde aber durch „neue“ subjektive Erfordernisse im Statut (etwa Wissen über begangene Kriegsverbrechen der Mitarbeiter) erschwert.

Menz Arbeit schließt mit einer Zusammenfassung der gewonnenen Erkenntnisse. Er weist auf die Problematik hin, dass Mitarbeiter privater Sicherheitsunternehmen ohne echte Verbindung zu einer Konfliktpartei nicht vom ICC sanktioniert werden können. Ihre Verbrechen müssten von nationalen Gerichten geahndet werden, die in der Folge der erhöhten Präsenz privater Akteure ohne Parteibindung in den kommenden Jahren überlastet werden würden. Menz begrüßt ferner die Forderungen einiger Wissenschaftler nach erweiterter Mitarbeiterüberwachung und weist auf die Praxisferne eines etwaigen Verbots privater Akteure im Statut hin. Armeen könnten ohne private Hilfe ihren Aufgaben nicht mehr nachkommen. Nochmals zeigt Menz prozessuale Probleme auf - zum Beispiel die Nachrangigkeit gegenüber nationalen Gerichten oder die Vielzahl staatlich gewährter Amnestien -, die dem Personal bisher eine faktische Straffreiheit gewährleistet haben. Diese müssten dringend behoben werden.

Vor dem Hintergrund, dass bisher eine Vielzahl von Staaten das Statut des ICC nicht ratifiziert und es bis heute keine Anklage gegen einen privaten Akteur gegeben hat, scheint Menz Analyse auf den ersten Blick realitätsfern. Er ist sich dessen durchaus bewusst, hätte dies jedoch deutlicher herausstellen müssen als mit der bloßen Feststellung, „dass sich der ICC in Zukunft mit einer derartigen Frage auseinanderzusetzen hat" (S. 24). Prinzipiell besticht Menz Arbeit sowohl durch eine klare Gliederung als auch eine überaus differenzierte Rechtsquellenbetrachtung. Die Einleitung erfüllt mit Darlegung von Problemstellung, Forschungsstand, Vorgehensweise und Methodik wissenschaftliche Ansprüche in hohem Maß, jedoch vermisst man als Leser hier eine exponiertere Darlegung der (widerlegten) Forschungsthese. Gut begründet scheint im Gesamtzusammenhang die Analyse des völkerrechtlichen Status privater Sicherheitsfirmen, die auf die Hauptuntersuchung im dritten Kapitel vorbereitet. Positiv hervorzuheben ist hier das Unterkapitel, das sich ausführlich dem nicht-internationalen Konflikt widmet; da dieser seit Ende des Kalten Krieg gehäuft auftritt, trägt Menz Ana- 
lyse den aktuellen Entwicklungen Rechnung. Zudem überzeugt die differenzierte Betrachtung der verschiedenen Untergruppen von Militär-/Sicherheitsfirmen. Zwischenergebnisse fassen innerhalb der gesamten Dissertation die gewonnen Erkenntnisse einprägsam zusammen und führen nachvollziehbar hin zum Endergebnis. Besonders gelungen ist das Hauptkapitel, das durch detailliert-differenzierte Prüfung der Voraussetzungen des Art. 8 ICC-Statut an Hand von Praxisfällen wie Abu Ghraib eindrucksvoll die These der völkerstrafrechtlichen Immunität widerlegt. Mit der zusätzlichen, gewissermaßen fakultativen Überprüfung der Verantwortlichkeiten des privaten Sicherheitsunternehmens und seiner Geschäftsführung zeichnet der Autor ein ausgewogenes Bild der aktuellen Rechtslage, jedoch scheint dies vor dem Hintergrund der auf die völkerstrafrechtliche Verantwortung der Mitarbeiter eingegrenzten Forschungsfrage überflüssig, wenn nicht sogar übereifrig. Insgesamt ist es sehr wahrscheinlich, dass Menz Arbeit bedeutenden Anteil an der wissenschaftlichen Debatte haben wird, die eventuell Druck auf Entscheidungsträger ausüben kann, sodass diese in künftigen Entscheidungen dem bisherigen Protektionismus gegenüber privatem Personal eine Absage erteilen.

\section{Carolin Deitmer}

\section{Gudrun Hentges, Hans-Wolfgang Plat- zer (Hrsg.), Europa - quo vadis? Ausge- wählte Problemfelder der europäischen Integrationspolitik, Wiesbaden (VS Ver- lag für Sozialwissenschaften) 2011.}

„Europa - quo vadis?“ - die Zukunft Europas steht zur Debatte. Der vorliegende Sammelband, herausgegeben von Gudrun Hentges und Hans-Wolfgang Platzer, baut vor allem auf die Aktualität seiner Themen. Laut Einleitung sehen die Herausgeber und Autoren allen voran in der Weltfinanz- und Eurokrise zentrale Herausforderungen für die europäische Politik: Wie schafft die EU eine rasche ökonomische Genesung angesichts der großen strukturellen Heterogenität seiner Mitgliedstaaten? Wie kann ein Demokratiedefizit bei der künftig notwendigen wirtschaftlichen Koordinierung vermieden werden? Und welche Instrumente braucht die EU, um solche Krisen zukünftig zu vermeiden? Wegen der Dringlichkeit dieser Fragen wird deshalb die Verortung der EU in ihrer vertraglichpolitischen Verfasstheit unter Berücksichtigung aktueller Entwicklungen als zentrales Ziel des Bandes angegeben.

Leider wird nur die Minderzahl der enthaltenen Artikel der eingangs formulierten Zielsetzung gerecht. Während Abschnitt zwei des Bandes (Weltwirtschaftskrise und Perspektiven der europäischen Wirtschafts- und Sozialpolitik) die beabsichtigte Ausrichtung gut trifft, und Kapitel eins (Politisch-institutionelle Perspektiven der Europäischen Union zwischen Vertiefung und Erweiterung) z.B. mit einer Diskussion der demokratischen Legitimität des Vertrags von Lissabon noch in dieselbe Stoßrichtung geht, hängen die übrigen Abschnitte und Kapitel des Bandes nur noch indirekt mit der Wirtschafts- oder Eurokrise zusammen. Die hier versammelten Beiträge über Arbeits- und Fluchtmigration sowie Rechtspopulismus und Rechtsextremismus begründen ihren Platz im Band nur durch die ebenfalls große Aktualität ihrer Themen. Exemplarisch sollen deshalb die Beiträge der beiden Herausgeber diskutiert werden, da sie die konzeptionelle Ambivalenz des Sammelbands gut widerspiegeln.

Der erste der beiden Beiträge trägt den Titel „Das Europäische Sozialmodell auf dem Prüfstand. Zur wissenschaftlichen Modelldebatte und den Perspektiven der Europäischen Sozialpolitik unter den Vorzeichen der Weltwirtschafts- und Eurokrise und des EU-Reformvertrags " und ist verfasst von Hans-Wolfgang Platzers. Dessen Absichten liegen erklärterweise in der Diskussion der Forschung zum Europäischen Sozialmodell, der Analyse der sozial-ökonomischen Herausforderungen in der Folge der Finanz- und Wirtschaftskrise sowie der Eurokrise, und abschließend in der Erörterung der jüngsten Entwicklungen und Perspektiven à la: „Quo vadis, Europäisches Sozialmodell?“. Nach einer Einleitung gibt Platzer einen Forschungsüberblick über die wissenschaftliche Literatur zum Europäischen Sozialmodell. Die Forschung teilt er in zwei Stränge: in einen komparativen Forschungszweig und eine integrationswissenschaftliche Debatte zur trans- und supranationalen Dimension des europäischen Sozialmodells. Haupt- augenmerk legt Platzer auf letztere, aus der er drei zentrale Folgerungen ableitet: Erstens seien zwar löbliche Fortschritte im Politikfeld der Antidiskriminierung erreicht worden, es sei aber, zweitens, durch eine forcierte Marktliberalisierung ein Wettbewerb der sozialpolitischen mitgliedstaatlichen Regeln an die Stelle des auf EU-Ebene sozialpolitisch regulierten Wettbewerbs getreten. Drittens und letztlich können die vermehrt eingesetzten „weichen“ OMK-Instrumente diesen Trend nicht auffangen. Vor diesem theoretischen Hintergrund untersucht Platzer nun noch in einem weiteren Kapitel wichtige „Politisierungsmuster", die für die Diskussion aktueller Ereignisse relevant sind, um dann zusammenfassend im letzten Abschnitt wie folgt zu schließen: Im Kontext aktueller Spannungen und Probleme sei der Ausbau einer supranationalen Sozialpolitik unwahrscheinlich, jedoch könne der Krisenstatus auch Chancen auf eine vorausschauende makroökonomische Koordination sein, die weit stärker als bisher auch Sozial- und Beschäftigungspolitik mit einbeziehen könnte. Insgesamt ist gegen die Konzeption des Beitrags Platzers grundsätzlich nichts einzuwenden, der Forschungsüberblick ist umfassend. Die Leserführung könnte aber noch verbessert werden. Vor allem eine prägnantere Trennung einzelner Ideen des Autors wäre wünschenswert gewesen.

Der zweite, hier vorgestellte Beitrag „Die extreme Rechte in Europa - zwischen niederländischem Rechtspopulismus und ungarischem Rechtsextremismus“ von Gudrun Hentges hängt hingegen nicht mit der Weltfinanz- oder Eurokrise zusammen. Er soll stattdessen der wachsenden Aktualität von Rechtspopulismus und Rechtsextremismus in Europa Rechnung tragen. Während die Autorin eingangs zwar beide Begriffe jeweils klar voneinander trennt, ist bis auf den Hinweis, dass sich der Beitrag vornehmlich den Einstellungen und Meinungen statt dem Verhalten der untersuchten Gruppen widmen soll, keine klare Fragestellung zu erkennen. So erschöpfen sich die beiden folgenden Kapitel des Beitrags in, erstens, einer rein deskriptiven Darstellung der Wahlerfolge europäischer rechtspopulistischer und rechtsextremer Parteien im Europaparlament seit 2001 und, zweitens, in der Extraktion der wichtigsten 
Programmpunkte der niederländischen PVV und der ungarischen Jobbik. (Die Auswahl genau dieser beiden rechtspopulistischen bzw. rechtsextremen Parteien soll sowohl West- als auch Osteuropa repräsentieren und wird jeweils durch deren besondere Wahlerfolge begründet.) Dem Leser erschließt sich jedoch der Zusammenhang der beiden Kapitel nicht. Der folgende Vergleich der Programmatiken der beiden vorgestellten Parteien ist nichts weiter als eine reine Gegenüberstellung ohne erkennbares Erkenntnisinteresse. Dem Leser bleibt nur, auf das abschließende Kapitel „Die wissenschaftliche Ursachendebatte und Gegenstrategien " zu hoffen, und hier besonders auf das Unterkapitel „Wissenschaftliche Erklärungsansätze“, das erstmals eine wie auch immer geartete wissenschaftliche Erkenntnis verspricht. Während nun also auf ca. drei Seiten eine „Erklärung der Ursachen “ versprochen wird, ist es dem Leser selbst überlassen, die Frage zu beantworten, wessen Ursachen überhaupt erklärt werden sollen - es ist wohl im weitesten Sinne die Rede von den Ursachen der Wahlerfolge rechtspopulistischer und rechtsextremer europäischer Parteien sowie der Verbreitung entsprechender Einstellungen und Meinungen in Europa. Hierfür bietet Hentges sozioökonomische und kulturelle Faktoren sowie eine Krise der Herrschaft an und diskutiert diese jeweils in ca. zwei Absätzen. Inhaltlich bleibt dieses Kapitel aber zu dünn und kann auch wegen der eher wenigen Verweise nicht als Forschungsüberblick gelten. Die abschließend vorgestellten „Gegenstrategien“ sind hingegen in ihrer einfachen Darstellung einem Schlusswort durchaus angemessen. Zusammenfassend kann Gudrun Hentges Beitrag nicht überzeugen. Zwar ist er so aktuell, dass zwischenzeitlich auch die deutsche Sarrazin-Debatte angeschnitten werden kann, ein sinnvolles Konzept, geschweige denn eine klare Fragestellung oder ein deutlich formuliertes Erkenntnisinteresse sind aber nicht erkennbar. Der Text erschöpft sich so größtenteils in der Deskription, um schließlich in einer viel zu knappen Diskussion der „Ursachen“ zu kapitulieren.

Alles in allem hinterlässt „Europa - quo vadis?“ einen gemischten Eindruck. Während die Qualität der Beiträge variiert, dabei durchaus Studenten wie For- scher gleichermaßen anspricht, ist die Konzeption des Sammelbands fragwürdig. An die eingangs erklärte Zielsetzung, die Herausforderungen der Weltfinanzoder Eurokrise für die EU zu diskutieren, hält sich maximal die Hälfte der mitwirkenden Autoren. Die restlichen Beiträge beziehen sich zwar auf aktuelle Themen, müssen den Herausgebern letztendlich jedoch das Zugeständnis entlocken, dass der Band doch eher allgemein versucht, „die mittlerweile breit gefächerten European Studies [zu] bereichern“ (S. 11).

Andres Hinrichsen

Georg Simonis, Helmut Elbers (Hrsg.), Externe EU-Governance, Wiesbaden (VS Verlag für Sozialwissenschaften) 2011.

Gerade im Hinblick auf die Analyse der Europäischen Union und der Gestaltung ihrer Außenbeziehungen greifen traditionelle Ansätze der staatenbasierten Außenpolitikanalyse nicht mehr. Wie aber kann man die Strukturen und Prozesse der Institutionalisierung und Koordinierung der Beziehungen der EU zu Drittstaaten fassen und untersuchen? Georg Simonis und Helmut Elbers bieten mit ihrem Lehrbuch „Externe EU-Governance“ einen anderen Ansatz an, der insbesondere auf der Vorstellung der Union als Staatenverbund und Governance-Regime beruht.

Die Autoren verfolgen mit ihrem Band drei Ziele (S. 16): Erstens die Entwicklung eines auf dem Governance-Ansatz fußenden analytischen Instrumentariums, zweitens die EU-Außenbeziehungen besser zu verstehen, dies insbesondere unter Berücksichtigung der normativen Basis der Union, und drittens die Darstellung unterschiedlicher Politikfelder und Fallstudien von externer EU-Governance.

Zunächst wird in einem ersten Teil das Governance-Konzept als analytisches Instrumentarium eingeführt und konkretisiert. „Governance“ wird hier als „Problembewältigung durch institutionell koordiniertes Handeln weitgehend unabhängiger politischer Akteure“ (S. 20) verstanden. Externe Governance ist dieser Lesart nach dann die Erweiterung dieser Form koordinierten Handelns über die Grenzen der EU hinaus. Bereits in diesem ersten Teil wird auch auf das Wesen der Union als Wertegemeinschaft und dessen Konsequenzen für externe EUGovernance Bezug genommen.

Im zweiten Abschnitt wird die Wertebasis der EU noch einmal im Detail beleuchtet. Was macht die EU als Akteur in den internationalen Beziehungen aus? Welche Kraft, welches Gestaltungspotenzial entfaltet sie? Die Autoren diskutieren hier vor allem die in der wissenschaftlichen Debatte etablierten Konzepte „Friedensmacht" und „normative Macht“, die zur Charakterisierung der EU in ihren Außenbeziehungen immer wieder herangezogen werden. In beiden Konzepten spielen die Werteorientierungen innerhalb der EU, ihre Tradition der Zivilgesellschaft eine wesentliche Rolle.

Diesen zwei eher konzeptionellen Teilen folgt im dritten Teil des Buches die Darstellung von vier Fallbeispielen, die alle im Kontext der EU-Nachbarschaftspolitik verortet sind.. Neben der externen Governance gegenüber den Ländern des Balkans werden die Beziehungen der EU zur Türkei und zur Ukraine sowie zu den Mittelmeeranrainern und zu Russland analysiert. In den vier Fällen wird jeweils die Frage aufgeworfen, inwieweit die normative Basis der EU tatsächlich geeignet ist, zu einer funktionierenden Governance und damit Gestaltung der Außenbeziehungen beizutragen.

Die Balkan-Governance ist ein gutes Beispiel, um die Möglichkeiten externer EU-Governance aber auch die Grenzen der EU als „normative Macht“ auf Staaten einzuwirken zu verdeutlichen. Die Länder des Westbalkan haben perspektivisch die Möglichkeit, Mitglied der EU zu werden. Mit Kroatien führt die EU bereits Beitrittsverhandlungen, Mazedonien und Montenegro besitzen Kandidatenstatus, Bosnien und Herzegowina sowie Albanien und Serbien befinden sich in einer Vorstufe. Wie das Beispiel zeigt, ist die normative Macht der EU dann besonders ausgeprägt, wenn die Drittstaaten ein großes Interesse an einer EU-Mitgliedschaft haben und den Bedingungen der EU entsprechend motiviert sind, einen Reformprozess einzuleiten. Allerdings zeigt sich, dass die normative Macht an ihre Grenzen stößt, sobald komplexe Probleme zwischen Drittstaaten auftreten (z.B. der Namensstreit zwischen Griechenland und Mazedonien). Des Weiteren weisen die Autoren auf die nicht unwesentliche Rolle der Bevölkerung in 
einem Partnerland hin: „Externe Governance durch die EU kann nur dann erfolgreich sein, wenn es in den Staaten der Region einen gesellschaftlichen Konsens bezüglich des EU-Beitritts und seiner Bedeutung gibt“ (S. 155).

Der vierte und letzte Teil beleuchtet vier funktional unterschiedliche Politikfelder, die Gegenstand externer EU-Governance sind: die Außenwirtschaftspolitik, die Entwicklungspolitik, die Migrationspolitik sowie die EU-Klimapolitik. Angesichts nationalstaatlicher Alleingänge im Bereich Migration und Grenzkontrolle so plante Dänemark zum Beispiel die Wiedereinführung von Grenzkontrollen; in den europäischen Hauptstädten herrscht Uneinigkeit darüber, wie mit den libyschen Flüchtlingen umgegangen werden soll - stellt sich allerdings die Frage, ob in diesem Politikfeld überhaupt von Governance gesprochen werden kann. Institutionell verankert sind immerhin ein gemeinsames europäisches Asylsystem und die gemeinsame Einwanderungspolitik. Die Arbeitsmigration verbleibt bis dato hingegen im Kompetenzbereich nationaler Regierungen.. Es wird sich zeigen, ob ein Konsens gefunden werden kann, diese auch auf europäischer Ebene zu regulieren, um somit zu einer weiteren Vergemeinschaftung der Migrations-Governance zu kommen.

Dem Lehrbuch „Externe EU-Governance“ gelingt es es, den Leser in die Thematik der externen Governance und in die damit zusammenhängenden grundlegenden Theorien und Konzepte einzuführen. Darüber hinaus verschafft es dem Lesereinen guten Überblick über die verschiedenen Perspektiven, aus denen die Europäische Union und die Gestaltung ihrer Außenbeziehungen betrachtet werden können. Die Fallstudien ermöglichen dem Leser die Auseinandersetzung mit und Analyse bzw. den Vergleich von einzelnen Regionen oder funktionalen Bereichen der externen Governance. Ein konkreterer Bezug der konzeptionellen Abschnitte auf die folgenden Fallbeispiele hätte die einzelnen Teile des Bandes noch besser miteinander verbunden und das Lehrbuch in seiner Gesamtheit abgerundet.

Anne-Rieke Stuhlmann
Michael Goodhart, Anja Mihr (Eds.), Human Rights in the 21st Century: Continuity and Change since 9/11, London (Palgrave MacMillan) 2011.

The post-9/11 human rights world: has everything changed? For better or worse? This is the question this anthology on human rights in the 21st century aims to answer - whether human rights have taken a turn for the worse, shown progress, or simply continued on into the future much as they were before this seminal event. This volume of thirteen articles encompasses a wide range of topics, regions, and viewpoints, expressed by academics coming from varied theoretical and practical backgrounds. From Russia, to the EU, to America, and the Netherlands, themes touched upon include the question of continuity or change in the international human rights regime, American exceptionalism, counter-terrorism, and torture. Themes particular to the post-9/11 human rights regime are also examined, such as terror blacklists, NGOs in the post-9/11 world, and business as an emerging actor in human rights regimes.

Three articles in particular will be examined in this review: Julie Mertus and Tazreena Sajjad's "The War on Terror through a Feminist Lens", which views post-9/11 human rights and the war on terror from a singular and interesting feminist viewpoint; Michael Goodheart's "Reverting to Form: American Exceptionalism and International Human Rights", which combines one of the most impactful countries regarding human rights with an important theme of this anthology, human rights exceptionalism; and finally Patricia Schneider's "Terror Blacklists on Trial: Smart Sanctions Challenged by Human Rights", which discusses contemporary human rights concerns in response to the post 9/11 creation of counter-terrorism measures such as terror blacklists.

In "The War on Terror through a Feminist Lens", Julie Mertus and Tazreena Sajjad apply a feminist framework of analysis to post-9/11 human theory and practice as it relates to the war on terror and, specifically, the role and state of women in Afghanistan. Their aim is to expose gendered ways of thinking, practices, assumption, and power dynamics in the war on terror as it relates to women's rights and status in Afghanistan. They attempt to do this by first offering an explanation and clarification of what it means to apply feminist theory to international relations issues. In this theoretical discussion the authors begin to digress slightly, and the theory unfortunately does not add much to the subsequent discussion. However, they then continue on to use two different lenses to examine the post-9/11 conflict in Afghanistan - as a war for women's human rights (a 'war for women') or as a 'war against women' and come to some valuable conclusions. By applying the lens of a 'war for women' they outline the way a lack of women's human rights was used as an excuse to invade Afghanistan as 'protectors of women's rights' with the burqua symbolizing the oppression they were combating. By applying the lens of a 'war against women' they show that once in Afghanistan, it became clear women's rights was not the main priority of the war, and indeed, when examined now, the little progress that Afghani women's rights made has already begun to diminish as US attention and motivation turns elsewhere.

Michael Goodheart's "Reverting to Form: American Exceptionalism and International Human Rights" challenges the conventional perspective that following 9/11, US policy changed dramatically in a way that trampled human rights. Goodheart incorporates a larger historical perspective into his analysis and concludes that the US human rights regime did not deteriorate following 9/11; it simply reverted back to its Cold War form. Goodheart states that the US has always fluctuated between a historically based "providential exceptionalism" (ie. Messianic exceptionalism), as evidenced in the Cold war and post-9/11, and a policy of pragmatic engagement, as evidenced in the 1990's when the US made great strides forward in the field of human rights. Goodheart concludes by examining current US policy, and finds that the picture painted by the Obama administration so far is mixed; while progress has been made (i.e. signaling the end of the "war on terror") the administration has also embraced flawed human rights policies. Goodheart's use of the historical approach to give 
weight to the concept of providential exceptionalism is a great strength in this article; subsequently, a meaningful analysis is the result. The article lacks only a fuller historical analysis extending to political/security decisions before the Cold War, to give what could be merely a coincidental pattern of the United States shifting from a messianic focus during the Cold War, to a pragmatic focus in the 90's, back to messianic method of action now real empirical weight and allow it to offer predictions for the future for the possible continuation of this pattern.

The third article of note, Patricia Schneider's "Terror Blacklists on Trial: Smart Sanctions Challenged by Human Rights" discusses contemporary human rights concerns in response to the post-9/11 creation of counter-terrorism measures such as 'smart sanctions', including terror blacklists. In the contemporary world, international law is changing - no longer restricted to states, it is coming to have a bearing on individuals as well, such as through the UN's controversial blacklisting and delisting procedures, or through state sanctions. Schneider takes an in-depth look at UN anti-terror sanctions, the much debated listing and delisting procedure, the European Court of Justice's decisions regarding listing/delisting, and EU terrorist lists vis à vis judgements before the European Court of Human Rights and national courts. Smart sanctions represent a new development in international law, affecting individuals and sometimes infringing upon their human rights; as such the empirical overview of methods and legal decisions regarding such smart sanctions is necessary and well received in this article. Schneider goes further, however, by ending the article by offering possible policy options and highlighting the need for effective, neutral review mechanisms available to those targeted by smart sanctions.

Overall, the articles in this anthology offer insightful perspectives and a dialogue on human rights change and continuity throughout the 21 st century. While one might have wished for a more coherent thematic selection of articles, the advantage of the variety in this book is that it offers many different viewpoints and frameworks from which to view contemporary human rights issues, constructing a dynamic, holistic picture of today's world.

\section{Savannah Carr-Wilson}

\section{Martin Kahl (Ed.), The Transnationalisa- tion of Risiks of Violence, Baden-Baden (Nomos Verlag) 2011.}

The emergence of transnational risks of violence - such as ethnic conflicts, organized crime, illegal immigration, or terrorism - is a relatively new phenomenon that challenges the perpetuation of peace and security in the 21st century. It can be regarded as a cause and a feature of the transition from the national to the post-national world. While states remain one source of risks of violence, sub-state actors play an increasing role in the destabilization of peace and security. Accordingly, a risk of violence is defined in this book as transnational "[...] when at minimum one non-state actor is a constitutive part of a conflictual cross-border relationship, which is endangering peace and security." (p. 7) Emphasizing that not every transnational risk necessarily leads to violent conflicts, Martin Kahl, the book's editor, reasons that the likelihood increases if identified risks are left to run their own course.

In the context of this "post-national constellation", The Transnationalisation of Risks of Violence deals with two central problems for the contemporary security situation: the growing weight of transnational risks of violence and the suitability of the strategies and instruments international organizations have adopted to manage these risks. This volume of the series Democracy, Security and Peace consists of seven contributions and is structured in three parts.

The first part of the book presents two conceptual frameworks for defining the phenomenon of transnational risks of violence in the post-national world and for examining its involvement in current conflicts. While Bernhard Zangl devises a conceptual framework without denominating this new paradigm - to explain the transformation of global security from the national to the post-national constellation, HansGeorg Ehrhart employs the theoretical conceptualization of security governance to grasp the transnationalisation of risks of violence.

The second part of the book reveals how transnational risks of violence find expression in concrete empirical cases: terrorism and regional conflict(s). Edwin Bakker analyzes the Al-Qaeda network as the most important example of jihadi terrorism by addressing the central question to what extent and under what conditions terrorism should be regarded as a transnational risk of violence. Marietta S. König focuses on the conflict in South Ossetia by asking to what extent it may be considered an appropriate example for the growing role of transnational actors, in perpetuating and increasing structures of violence.

The third part of the book deals with the management or governance of transnational risks by international organizations. Monika Heupel investigates whether the United Nations Security Council has developed a legitimate and effective response to the different challenges posed by transnational security problems. Jörg Monar examines the European Union, which has emerged as an "[...] actor in the sphere of international risk management in its own right" since 1999 (p. 121). Finally, Wolfgang Zellner scrutinizes the concepts, strategies and instruments the OSCE has adopted to cope with transnational risks of violence.

This book provides a sound and balanced overview of the phenomenon of the transnationalisation of risks of violence. It ties in with existing social constructivist accounts about the threat constellation in the contemporary postnational world. The clear, coherent and logical structure enables the reader to easily follow the volume's underlying rationale, not least due to the very detailed and explicit introduction: Martin Kahl summarizes each article by not only providing a synopsis of the main arguments but also by presenting the respective findings. The application to concrete empirical examples, in the second part of the book, contributes to a more differentiated understanding of this emerging phenomenon. On the one hand, Edwin Bakker comes to the conclusion that current forms of terrorism, and the case of the AlQaeda network in particular, can be 
taken as unambiguous examples of a transnationalisation of risks of violence. On the other hand, Marietta S. König's findings show that transnational risks and threats provide only a sample of the general conflict situation in cases such as South Ossetia. The findings in the book are rather disparate, making it difficult to come to a general conclusion. In that way, the reader is given the possibility to draw his/ her own inference. Nevertheless, Martin Kahl provides a brief upshot in which he recapitulates that today's transnational risks of violence are multifaceted, i.e. they occur in multiple forms, have various causes and different characteristics. Given the extent of this book, Martin Kahl is well aware of the fact that further analysis remains necessary and opens herewith a field of future research.

The book is very comprehensible and still offers thought-provoking impulses. Therefore, it is worth reading for both active researchers in the field of transnational risks of violence as well as a broad readership interested in this topic.

Anika Kirchner

\section{Michael Brzoska, Hans-Georg Ehrhart and Jens Narten, Multi-Stakeholder Security Partnerships: A Critical Assess- ment with Case Studies from Afgha- nistan, DR Congo, and Kosovo, Baden- Baden, (Nomos Verlag) 2011.}

Multi-Stakeholder Partnerships (MSPs) are defined by three characteristics: they must include representatives at the international, national, and subnational level, they must represent "true partnership" (p. 7) with resources invested over long periods of time and the investors must have a common aim or objective to pursue within the MSP. This book cites that while there is much research on MSPs, up to this point the research on security-related MSPs is lacking. Therefore, through several articles, this book examines the role of, success and importance of MSPs in the security sector and in post-conflict situations (MSP-SEC). The introduction does a nice job of outlining the overall framework of the book in a comprehensive way. The book is divided into three sections. The first deals with defining MSPs and their importance in the larger scope of globalization and security. Section two deals with overarching themes in MSPs and Section three looks at three case studies.

In the first section, chapter one does a nice job of explaining MSPs and discussing the remaining problems with MSP-SECs. Here the idea of "freedom from fear" vs. "freedom from want" is introduced and mentioned often throughout the book. Many MSP-SECs still focus mostly on the fear aspect and have yet to really promote positive peace. One thing that may have strengthened this section would have been the use of real examples of MSPs throughout the chapter to illustrate more clearly the existing problems . Chapter 2 thoroughly outlines the new international system and how, with the emergence of new actors in the system, it is necessary to include new actors in security policy. This chapter, however, only mentions MSPs at the end, in terms of their place within security governance, going on to explain what MSPs are. This chapter would therefore possibly be more effective as the first chapter in the book, in order to first place MSPs within the global governance system before defining and analyzing them.

Section two looks at different themes within MSPs and Chapter three begins by looking at MSPs and disarmament, demobilization, and reintegration (DDR) and small arms and light weapons (SALW). This chapter stresses the important role of MSPs not just in regulating but in building new norms. Here it is where the importance of local ownership comes into play. MSPs are trying to institutionalize local partners and get individuals and communities to give up personal security for the good of larger security. This is not easily done purely through regulation. This chapter, as well as the other chapters in this section, does a good job of illustrating the problems and difficulties through many examples, in this case gender and weapons. Chapter four does this as well by identifying the types of MSPs in post-conflict situations also with helpful examples, stressing the need to bridge the gap between the government and the people in post-conflict or failed states, because this bridge is often weak. Through community or local participation in decision-making, this can be somewhat accomplished. However, this chapter also introduces the fact that local ownership can be very dangerous if not well monitored and maintained and that it can lead to power grabs, which is also illustrated in the case studies. For this reason international groups can play a very important monitoring role in post conflict MSPs. Chapter five identifies the need for MSPs in SSR. Stating that "SSR needs to be 'owned' by local actors both for practical reason and because of its need for international legitimacy" (92). But a question raised here, which will become evident in the case studies, is if the various actors actually have a shared objective. When looking at MSP-SECs, actors in such roles as military, policy, judiciary, and local all come together but most likely under different motivations and with different goals.

These three chapters summarize very well the thematic areas where MSP-SECs can be useful with illustrative examples and clear definitions. These themes and the difficulties MSPs encounter remain clearly present throughout the three case studies in section 3 . The importance of this section or the reasons for including these particular three themes, therefore, is easily recognized.

Chapter six analyzes in detail the Kosovo Protection Corps Resettlement Program (KPC-RF) as the first of three MSP case studies in section three. The chapter explains early on exactly why KPC-RF qualifies as a MSP, which is also done in the other two sections and is very useful in connecting section one with section three. A survey was conducted asking actors from all three levels (international, national and local) about the success of the program. These results showed somewhat mixed messages. Also the lack of a balance in power between the levels was discussed. The partnership is still heavily seen as top-down. Furthermore, the success of the program and also its effects on peacebuilding and human security were seen much more positively by the international actors being interviewed than the national or local ones. One relationship that is not heavily discussed in the chapter is why the groups with the most power would also be the groups that find the program largely successful while others do not. 
This would have been interesting to add to the discussion, but was not heavily touched upon.

Chapter seven takes the case study of the DR Congo and the ILSC (Initiatives Locales de Sécurité Communautaire). It involves a field study carried out in the Ituri region. The chapter does a really good job not only of explaining the context of the situation in the DRC and the cultural characteristics that make the MSP structure the way it is, but also of classifying the goals and impacts of ILSC as well as the jobs of each actor. They also explain which possible partners are missing from the MSP, which is useful to the reader. The chapter also illustrates the weaknesses of the actors involved, especially the NKs (the community level unit) and the conflicts between the state and the NKs. Overall it does a good job of explaining the strengths and weaknesses of an MSP in a rural and conflict prone area.

Chapter eight is the final case study of Afghanistan and the Disbandment of Illegal Armed Groups (DIAG). They provide a comprehensive table describing the actors and ownership of DIAG and give a detailed history of DDR in Afghanistan (being mostly top down) and about the development of DIAG, which gives a clear context for the environment in which the MSP is working. DIAG is the only Afghan government-run MSP-SEC. However, in reality the international actors still play a large roll, and at the beginning, involving local Afghans was difficult. The article comments extensively on the organizational and strategic problems that occur between actors and have hindered effectiveness. Also some corruption issues in arms gathering and the outdated policies of collection are mentioned. Additionally, there remains a lack of trust between the people and the government, which makes disarming the population difficult. In general, they site that DIAG is a strong and successful process that is unsuccessful because of government problems and that there is a contradiction between the reports of DIAG success and the situation on the ground.

In general, the case studies were very well chosen, representing three different areas of the world and three different conflict or security reform contexts.
The individual MSPs chosen were very suitable for a short case study that could be discussed in detail within the confines of this book, as they were concentrated in purpose while still relating overall to the security sector. The structure of the chapters as well helped to relate the case studies to the MSP characteristics and flaws discussed in section 1 and the themes of section two. The case studies did not shy away from pointing out the many remaining difficulties and negative aspects of the programs. A common theme throughout was the lack of a common objective or a common idea of what "success" is between the actors. This points to the importance of on-theground qualitative evaluations and not only having success defined by those at the top. The book concludes by rehighlighting the importance of MSP-SECs for peacebuilding and human security and gives very specific recommendations to external stakeholders, especially the EU. Overall the book is an instructive first attempt at looking critically at the role and success of MSPs in post-conflict and security reform situations. The willingness to point to both strengths and weaknesses of the MSP structure lends legitimacy to the arguments and leaves the reader knowing that there is much more work to be done, but the inclusion of all involved actors is a step in the right direction.

Caitlin Corrigan

\section{Ein bemerkens- wertes Buch}

Peter Brinkmann, www.european-circle.de Mai 2010

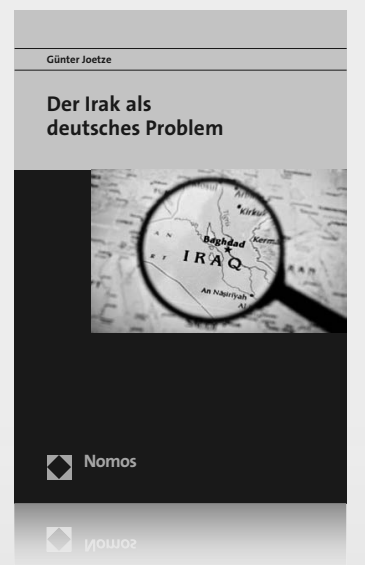
Der Irak als deutsches
Problem

Von Günter Joetze

2010, 310 S., brosch., 29,-€

ISBN 978-3-8329-5365-2

Der Irak-Krieg und der deutsche Widerspruch hielten unsere Öffentlichkeit ein Jahr lang in Bann. Noch nie war die Bundesrepublik solchen Polemiken ihres Hauptverbündeten ausgesetzt. Auch sie trägt noch heute an den Folgen für die internationale Ordnung. Um diesen Problemen nachzugehen, sichtete Dr. Günter Joetze seit 2002 amtliches Material und befragte Zeitzeugen.

"bislang beste Darstellung des transatlantischen Zerwürfnisses um den Kampf gegen Saddam Hussein."

Hans Monath, www.tagesspiegel.de November 2010

www.nomos-shop.de/12257

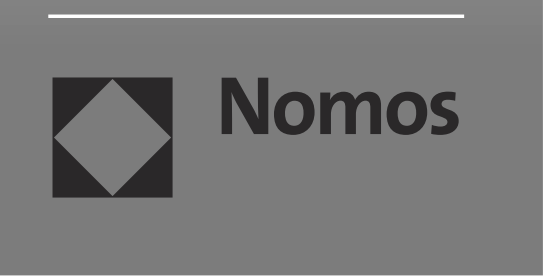

